\title{
Article \\ Deficiency in Tissue Non-Specific Alkaline Phosphatase Leads to Steatohepatitis in Mice Fed a High Fat Diet Similar to That Produced by a Methionine and Choline Deficient Diet
}

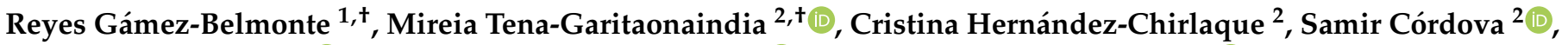 \\ Diego Ceacero-Heras ${ }^{2}{ }^{\mathbb{D}}$, Fermín Sánchez de Medina ${ }^{1, *(1)}$ and Olga Martínez-Augustin ${ }^{2}$ (I) \\ 1 Department of Pharmacology, CIBERehd, School of Pharmacy, Instituto de Investigación Biosanitaria \\ ibs.GRANADA, University of Granada, 18071 Granada, Spain; \\ MariadelosReyes.GamezBelmonte@uk-erlangen.de \\ 2 Department of Biochemistry and Molecular Biology 2, CIBERehd, School of Pharmacy, Instituto de \\ Investigación Biosanitaria ibs.GRANADA, Instituto de Nutrición y Tecnología de los Alimentos José Mataix, \\ University of Granada, 18071 Granada, Spain; mireiat94@gmail.com (M.T.-G.); \\ cristinahech@hotmail.com (C.H.-C.); clamirja25@correo.ugr.es (S.C.); diegocastulo98@gmail.com (D.C.-H.); \\ omartine@ugr.es (O.M.-A.) \\ * Correspondence: fsanchez@ugr.es; Tel.: +34-958-241747 \\ + Both authors contributed equally to this work.
}

Citation: Gámez-Belmonte, R.; Tena-Garitaonaindia, M.; HernándezChirlaque, C.; Córdova, S.; CeaceroHeras, D.; de Medina, F.S.; MartínezAugustin, O. Deficiency in Tissue NonSpecific Alkaline Phosphatase Leads to Steatohepatitis in Mice Fed a High Fat Diet Similar to That Produced by a Methionine and Choline Deficient Diet. Int. J. Mol. Sci. 2021, 22, 51. https://dx.doi.org/10.3390/ ijms22010051

Received: 27 November 2020 Accepted: 21 December 2020

Published: 23 December 2020

Publisher's Note: MDPI stays neutral with regard to jurisdictional claims in published maps and institutional affiliations.

Copyright: () 2020 by the authors. Licensee MDPI, Basel, Switzerland. This article is an open access article distributed under the terms and conditions of the Creative Commons Attribution (CC BY) license (https: / / creativecommons.org/ licenses/by/4.0/).
Abstract: The liver expresses tissue-nonspecific alkaline phosphatase (TNAP), which may participate in the defense against bacterial components, in cell regulation as part of the purinome or in bile secretion, among other roles. We aimed to study the role of TNAP in the development of hepatosteatosis. $\mathrm{TNAP}^{+/-}$haplodeficient and wild type (WT) mice were fed a control diet (containing 10\% fat $w / w$ ) or the same diet deficient in methionine and choline (MCD diet). The MCD diet induced substantial weight loss together with hepatic steatosis and increased alanine aminotransferase (ALT) plasma levels, but no differences in IL-6, TNF, insulin or resistin. There were no substantial differences between $\mathrm{TNAP}^{+/-}$and $\mathrm{WT}$ mice fed the $\mathrm{MCD}$ diet. In turn, $\mathrm{TNAP}^{+/-}$mice receiving the control diet presented hepatic steatosis with alterations in metabolic parameters very similar to those induced by the MCD diet. Nevertheless, no weight loss, increased ALT plasma levels or hypoglycemia were observed. These mice also presented increased levels of liver TNF and systemic resistin and glucagon compared to WT mice. The phenotype of $\mathrm{TNAP}^{+/-}$mice fed a standard diet was normal. In conclusion, TNAP haplodeficiency induces steatosis comparable to that produced by a MCD diet when fed a control diet.

Keywords: methionine and choline diet; metabolic syndrome; biliary acids; tissue non-specific alkaline phosphatase; steatohepatitis

\section{Introduction}

Non-alcoholic fatty liver disease (NAFLD), defined by the accumulation of fat in the liver in the absence of significant alcohol intake, encompasses a spectrum of liver diseases that can be further characterized as either non-alcoholic fatty liver (NAFL) or non-alcoholic steatohepatitis (NASH), based on the presence or absence of inflammation and liver cell injury. Because of its high incidence/prevalence, and its possible progression to cirrhosis and ultimately to hepatic cancer, NAFLD is increasingly attracting attention [1]. In addition, there is a close link between metabolic syndrome and NAFLD [2].

Alkaline phosphatases are a group of isoenzymes that in humans include the intestinal (IAP), tissue non-specific (TNAP), fetal and placental isoforms [3]. These enzymes share substrates and a common mechanism of action. Among them, TNAP comprises three isoforms (liver, bone and kidney), identical in their final sequence but differing in their glycosylations. TNAP is a pleiotropic enzyme expressed in multiple cell types and 
tissues, including liver, bone, brain, intestine, lymphocytes, macrophages or neutrophils. Multiple substrates, such as bacterial components (LPS), nucleotides, phosphorylcholine (PC), phosphoryl-ethanolamine or pyridoxal-5'-phosphate, have been described for TNAP, and as a consequence, it has a role in multiple systemic processes, including bone mineralization, vitamin $\mathrm{B}_{6}$ metabolism and neurogenesis [4]. Mutations in the encoding gene ALPL are the cause of hypophosphatasia.

In recent years, TNAP has been shown to exert different anti-inflammatory effects depending on its actions on the innate and adaptive immune system. Two main mechanisms of action have been described. An increase in the production of IL- 6 and TNF has been observed in neutrophils from heterozygous $\mathrm{TNAP}^{+/-}$mice stimulated with LPS, indicating an anti-inflammatory effect of the enzyme, possibly dependent on LPS dephosphorylation [5]. Another mechanism for TNAP to influence inflammation is the regulation of purinergic signaling. In this regard, a recent study shows that TNAP inhibition in neutrophils significantly exacerbates ATP-associated activation and secretion of IL-1 $\beta$ [6]. We have shown that TNAP is required for T cell activation and $\mathrm{TNAP}^{+/-} \mathrm{T}$ cells exhibit a decreased colitogenic potential in vivo [7].

NAFLD and other features of metabolic syndrome, including obesity or diabetes, are linked to subclinical inflammation, induced by alterations in the intestinal barrier, resulting in increased levels of circulating bacterial antigens. Several studies have shown the association of IAP with metabolic syndrome. In this regard, the administration of IAP to mice fed a high-fat diet prevents endotoxemia and the associated metabolic syndrome [8], while Akp3 (duodenal AP) knock-out mice show faster weight gain [9], visceral fat accumulation and hepatic steatosis, as well as insulin resistance. Similarly, IAP deficiency has been related to a higher lipid absorption in animals fed a high-fat diet [8]. The effects of IAP on metabolic syndrome have been attributed to its ability to dephosphorylate LPS and possibly other bacterial molecules. Since TNAP shares anti-inflammatory actions and mechanisms with IAP and is involved in purinergic signaling, we hypothesized that it could have a role in preventing hepatosteatosis. TNAP KO mice are only viable for a few days; they die before weaning and show vitamin $\mathrm{B}_{6}$-sensitive epilepsy and impaired bone mineralization. $\mathrm{TNAP}^{+/-}$haplodeficient mice are, however, viable, although they exhibit an altered immune phenotype as noted.

TNAP is also known to dephosphorylate PC, to obtain choline to be absorbed in the intestine, and is needed for the synthesis of phospholipids, acetylcholine and trimethylglycine. Choline deficiency inhibits the synthesis of phosphatidylcholine required for very low-density lipoprotein (VLDL) production and induces lipid accumulation in the liver $[10,11]$. Here we explore the effect of the absence of an allele of TNAP (i.e., TNAP haplodeficiency) on the development of fatty liver by methionine- and choline-deficient $\operatorname{diet}(\mathrm{MCD})$ with a $10 \% w / w$ content of fat $(22.1 \% \mathrm{Kcal}$ from fat). In turn, the deficiency of methionine decreases the biosynthesis of glutathione, leading to oxidative stress, which in turn contributes to liver damage [12]. We found that $\mathrm{TNAP}^{+/-}$responded to the $\mathrm{MCD}$ diet just like WT mice but developed fatty liver with the control diet, underscoring a role of TNAP in the susceptibility to this dietary challenge.

\section{Results}

\subsection{The Absence of a Single Alpl Allele Does Not Modify the Response of Mice to the MCD Diet}

As expected, feeding the MCD diet resulted in sustained body weight loss in wild-type mice for up to 17 days, when the experiment was halted for ethical reasons, as MCD-fed mice had lost more than $20 \%$ of their body weight (Figure 1A). At this time, the expected development of fatty liver was confirmed, as shown by steatosis (Figure 1B,C) and increased alanine aminotransferase (ALT) circulating levels (Figure 1D). Nevertheless, no alteration in the expression of pannexin 1 (Panx1, recently related to inflammation in non-alcoholic steatohepatitis and to liver damage [13]) or the fibrosis markers transforming growth factor $\beta 1$ (Tgfb1) or desmin (Des) was observed (Figure 2). Accordingly, no hepatic inflammation was shown, since liver mRNA levels of IL-1 $($ (Il1b), IL-6 (Il6), TNF (Tnf) and osteopontin 
(Spp1) were comparable to those of WT mice fed the control diet (Figure 2). The hepatic expression of other inflammation-related markers, LPS binding protein (Lbp), Cd14 or glutathione peroxidase 1 (GPx1), was also unchanged (Figure 2). Interestingly, alkaline phosphatase activity and its sensitivity to levamisole were increased in WT mice fed the MCD diet, consistent with a change in isoform (Figure 3). In general, these results indicate the induction of steatosis but no inflammation in the liver of WT animals fed an MCD diet.

A.

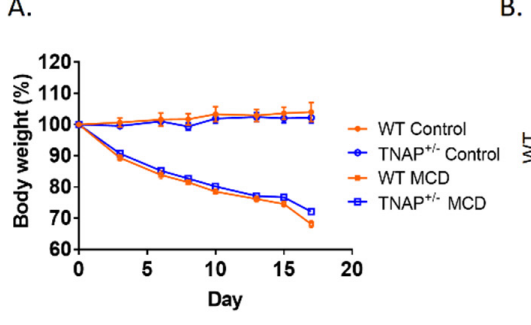

D.

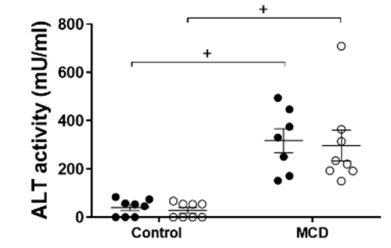

B.
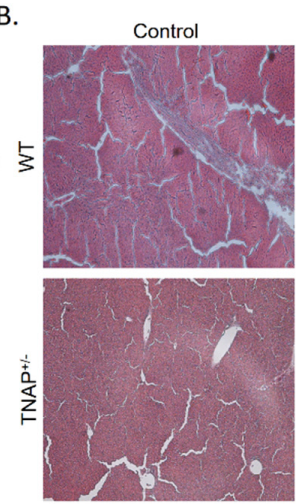

E.

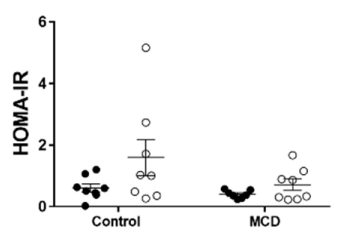

C.
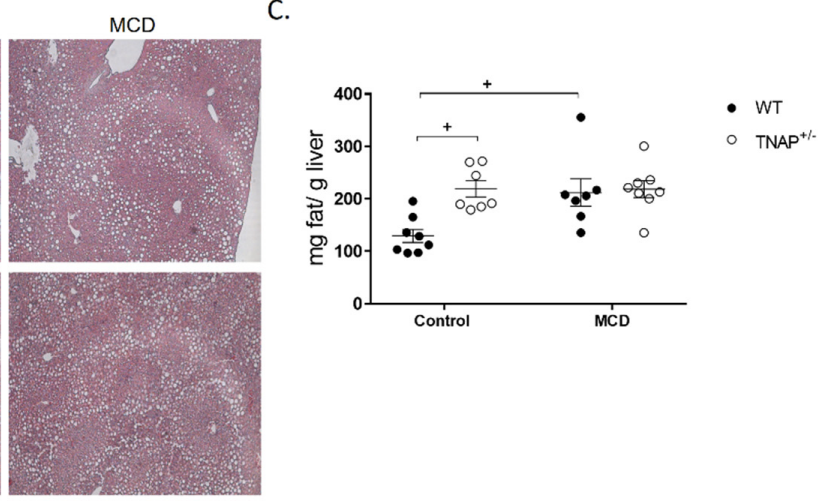

F.

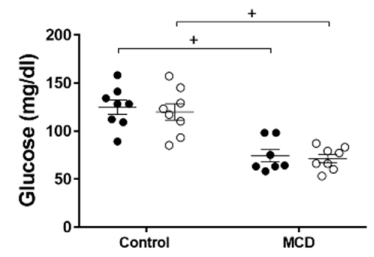

Figure 1. Main parameters of the methionine- and choline-deficient diet (MCD) model. Body weight evolution (A), liver histology (B), fat liver content (C), plasma ALT activity (D), glucose concentration in plasma (E) and HOMA-IR index (F) of wild type (WT) and TNAP heterozygous mice (TNAP $\left.{ }^{+/-}\right)$fed a control diet $(10 \% w / w$ fat, control) or the same diet deficient in choline and methionine $(\mathrm{MCD})^{+} p<0.05$.
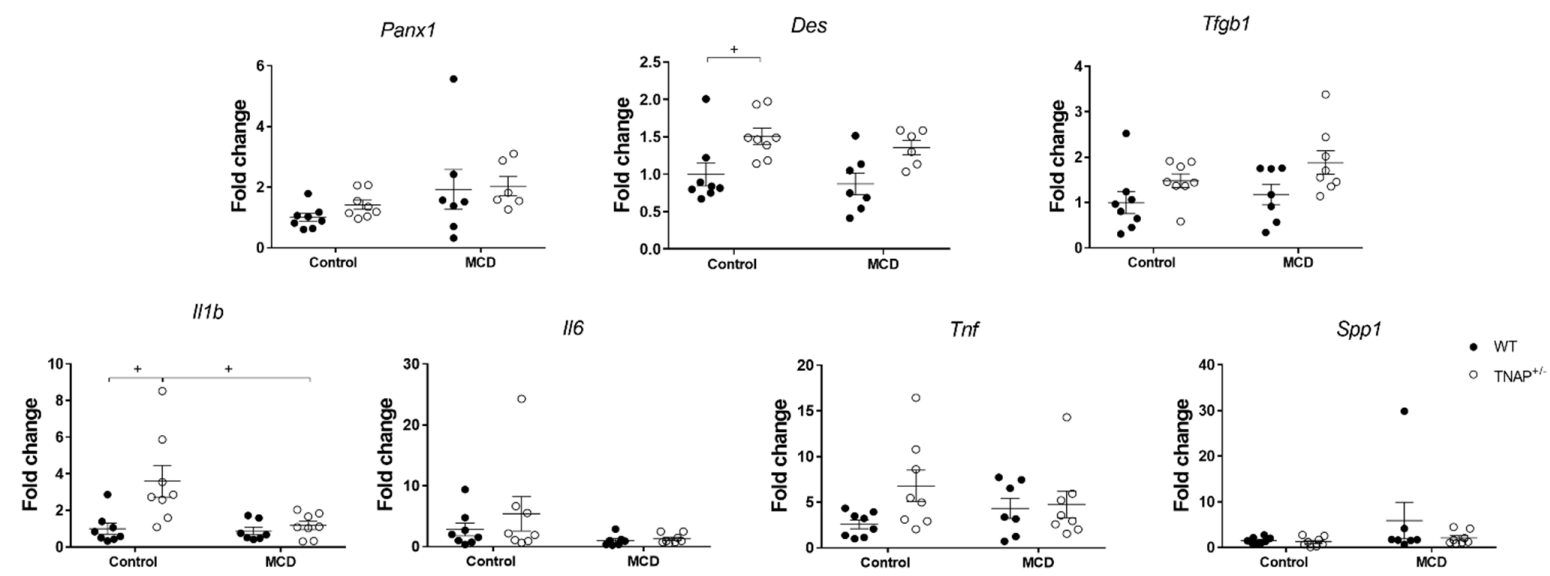

Lbp

Cd14
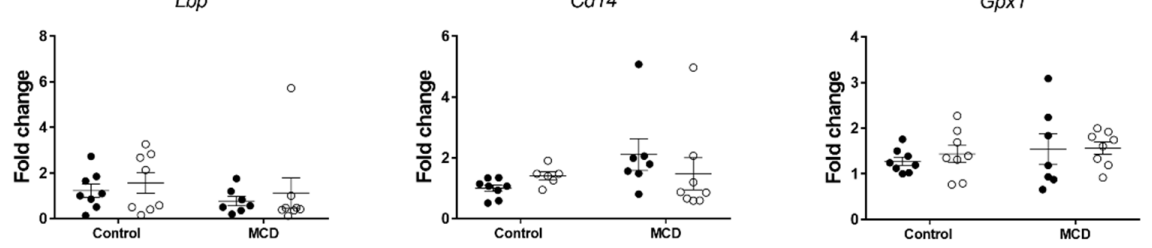

Figure 2. Hepatic expression of fibrosis and inflammatory genes in wild-type (WT) and TNAP heterozygous mice (TNAP /- ) a control diet $(10 \% w / w$ fat, control) or the same diet deficient in choline and methionine (MCD). Fold change of qRT-PCR data are shown. ${ }^{+} p<0.05$. 

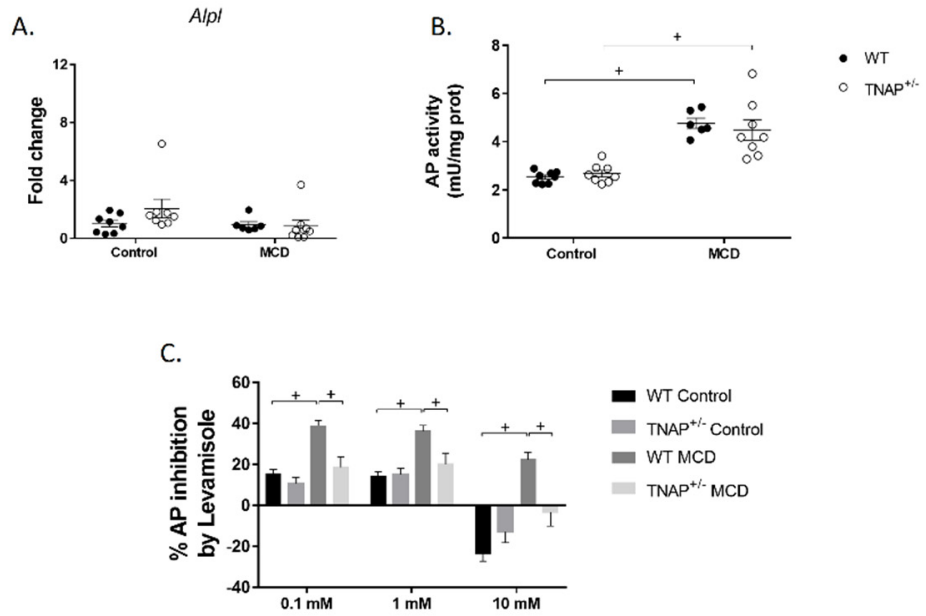

Figure 3. Hepatic alkaline phosphatase expression (A), activity (B) and sensitivity to levamisole (C) of wild-type (WT) and TNAP heterozygous mice (TNAP $\left.{ }^{+-}\right)$fed a control diet $(10 \% w / w$ fat, control) or the same diet deficient in choline and methionine (MCD). For AP expression, fold change qRT-PCR data are shown. ${ }^{+} p<0.05$.

Glucidic metabolism genes were also assessed. No changes in the expression of glucose-6-phosphatase catalytic subunit (G6pc) or glucose transporter 2 (Glut2) were observed in WT mice fed the MCD diet, while a deep inhibition of liver phosphoenolpyruvate carboxykinase 1 (Pck1), the key gluconeogenic enzyme, as well as induction of pyruvate dehydrogenase kinase 4 (Pdk4), were noted (Figure 4). Pyruvate dehydrogenase kinase 4 inhibits the pyruvate dehydrogenase complex and therefore the synthesis of acetyl-CoA from pyruvate.
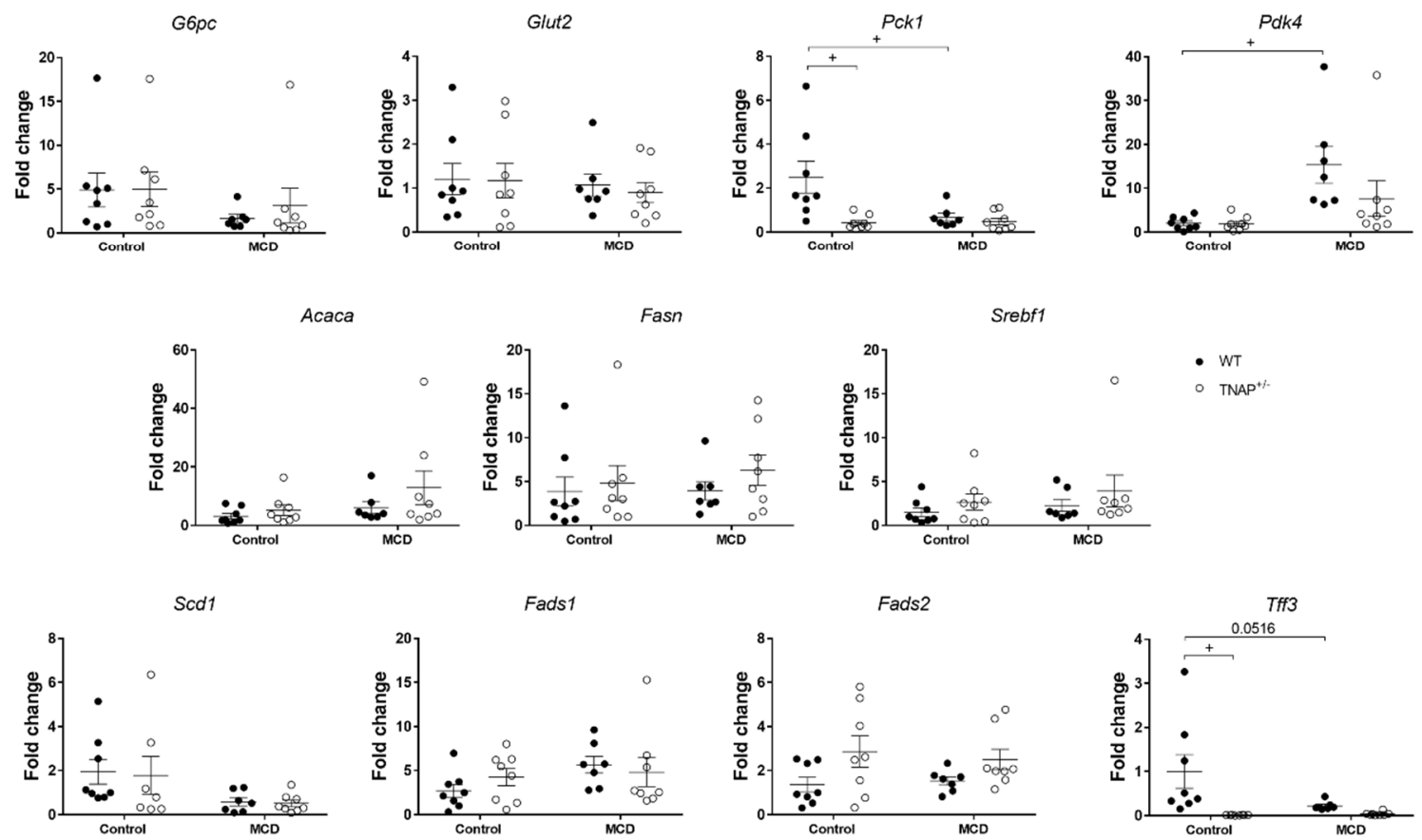

Figure 4. Hepatic expression of glucidic and lipidic metabolism genes in wild-type (WT) and TNAP heterozygous mice $\left(\mathrm{TNAP}^{+/-}\right)$fed a control diet $(10 \% w / w$ fat, control) or the same diet deficient in choline and methionine (MCD). Fold change of qRT-PCR data are shown. ${ }^{+} p<0.05$.

When liver fatty acid metabolism was studied, the expression of key enzymes in fatty acid synthesis, namely acetyl-CoA carboxylase (Acaca) and fatty acid synthase (Fasn), 
of the transcription factor steroid regulatory element-binding protein 1 (Srebf1), and of fatty acid desaturase genes (Scd1, Fads1 or Fads2), was unchanged (Figure 4). Nevertheless, an almost significant decrease in Tff3 $(p=0.056)$, recently described as a regulator of lipid metabolism [14], was observed (Figure 4).

The MCD diet induced hypoglycemia with no change in insulin levels; therefore, the HOMA-IR index was not affected (Figures 1E and 5). Plasma levels of leptin, IL-6, C-peptide, ghrelin, gastric inhibitory peptide (GIP), pancreatic peptide (PP), peptide YY (PYY), resistin and amylin were also unchanged (Figure 5). Only the levels of glucagon-like peptide 1 (GLP-1) were shown to be increased, while those of glucagon were reduced, just short of significance $(p=0.08)$.
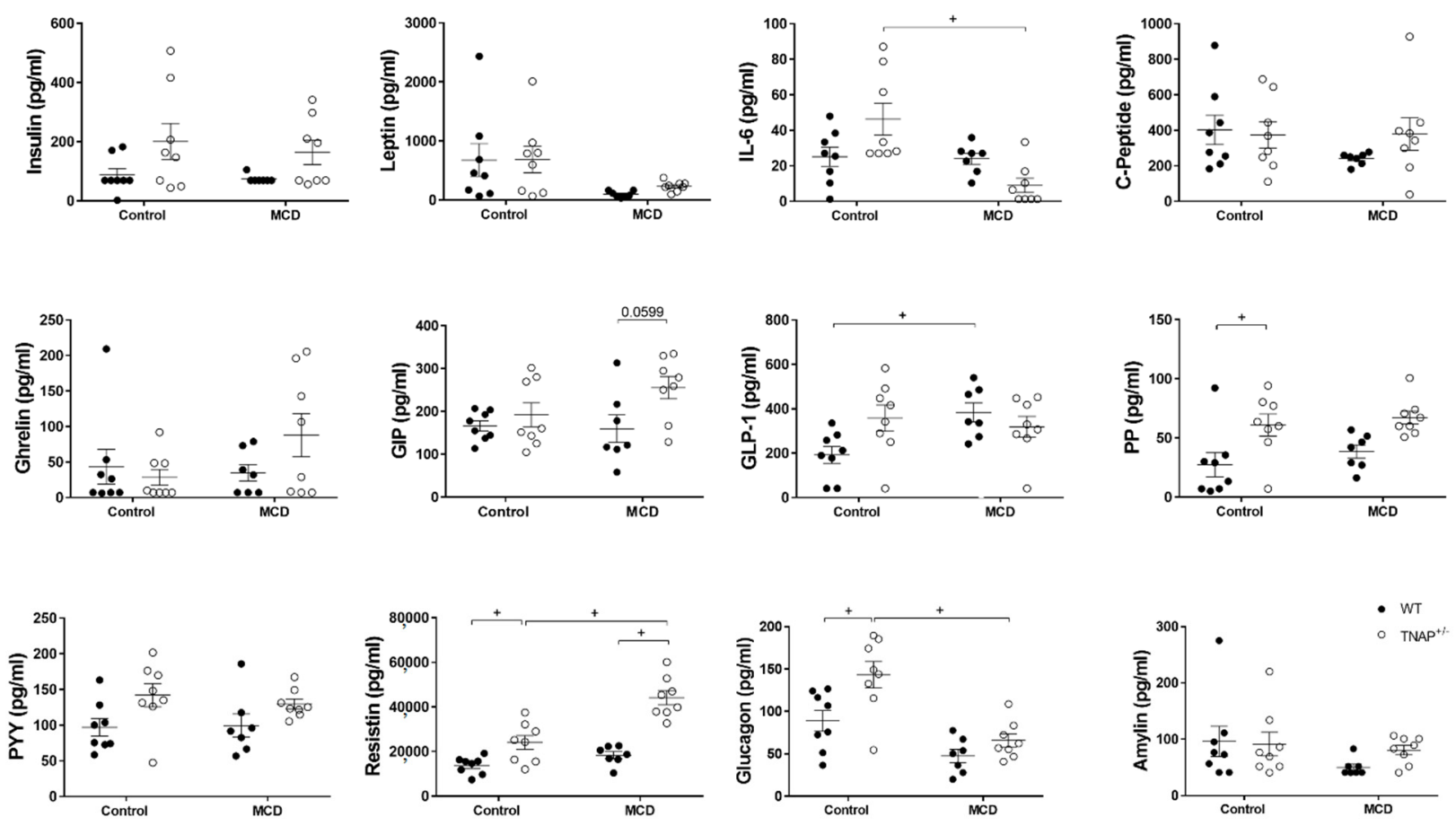

Figure 5. Plasma concentration of inflammation-, obesity- and steatosis-related markers in wild-type (WT) and TNAP heterozygous mice $\left(\mathrm{TNAP}^{+/-}\right)$fed a control diet $(10 \% w / w$ fat, control) or the same diet deficient in choline and methionine (MCD). Results from the Metabolic Hormone Expanded Panel multiplex are shown. ${ }^{+} p<0.05$.

The assessment of intestinal parameters showed that weight-to-length ratio was decreased in the colon, ileum and jejunum. Neither alkaline phosphatase activity nor the expression of Alpl (encoding TNAP) were altered by the administration of the MCD diet (Figure S1A/B). Only, as described in liver, sensitivity to levamisole was augmented. Intestinal inflammatory or tight junction genes such as S100a8, Cldn4 and Tjp1 remained unchanged (Figure S1C/D).

The effects of the MCD diet on $\mathrm{TNAP}^{+/-}$mice were very similar to those on wild type mice, and the phenotypes were almost indistinguishable. The exception to this rule was the increased levels of resistin and an almost significant increase in GIP $(p=0.06)$ in the plasma of $\mathrm{TNAP}^{+/-}$mice (Figure 5), plus the lower sensitivity to levamisole of alkaline phosphatase both in the intestine and liver, which was very similar to that of control-diet-fed groups (Figure 3C).

\subsection{Alpl Haplodeficiency Induces Hepatic Fat Accumulation in Mice Fed a Control Diet}

Somewhat unexpectedly, when fed the control diet, the phenotype of $\mathrm{TNAP}^{+/-}$ mice differed considerably from that of the WT group. Thus systemic levels of glucagon, resistin, GLP-1 and PP were increased in the $\mathrm{TNAP}^{+/-}$group (Figure 5). Insulin and PYY showed a similar pattern but without reaching significance. An almost significant systemic 
increase in IL-6 ( $p=0.076)$ was also observed. Remarkably, liver fat was augmented in TNAP haplodeficient mice and was comparable to that of MCD-diet-fed animals of both genotypes (Figure 1). However, steatosis presented with microdeposits at the histological level, while larger fat deposits were observed in mice given the MCD diet with either genotype (Figure 1B). In turn, there was no change in body weight, fasting blood glucose, insulin or HOMA index, although values tended to be higher in $\mathrm{TNAP}^{+/-}$mice in the latter case (Figures 1 and 5).

This phenotype was not anticipated, since $\mathrm{TNAP}^{+/-}$mice show no obvious phenotype at the hepatic level, or indeed globally, in basal conditions. Since the control diet used includes a higher-than-normal fat content, we decided to analyze the hepatic status under standard feeding conditions, i.e., using a regular chow diet. In these conditions, no steatosis was observed (Figure S2A), indicating that the interaction between genotype and the control diet is responsible for the observed phenotype.

\subsection{Characterization of Steatosis in Alpl Haplodeficient Mice}

In addition to steatosis, RT-qPCR analysis revealed an increased expression of hepatic $I l 1 b$ and an almost significant increase in $\operatorname{Tnf}(p=0.08)$. No other inflammation-related genes were affected (Il6, Lbp, Cd14, Spp1 or Gpx1) (Figure 2). In turn, desmin gene expression was increased in the liver of haplodeficient mice, consistent with possible fibrosis, and Tgfb expression exhibited a very similar pattern, without reaching significance. Plasma ALT activity and Panx1 mRNA level were similar to those of WT mice fed the same diet (Figures 1D and 2). Taken together, these results are consistent with mild inflammation with no substantial hepatic injury.

Next, the expression of key genes involved in hepatic glucidic and fatty acid metabolism was studied using RT-qPCR. As in the mice fed the MCD diet, Pck1 and Tff3 expression were significantly and profoundly decreased (Figure 4). We next looked at the expression of acyl-Coenzyme A synthetases and acyl-CoA thioesterases, which regulate fatty acid metabolism, namely Acsm2, encoding mitochondrial acyl-coenzyme A synthetase, and Acot5, encoding an acyl-coenzyme A thioesterase (Figure 6). The expression of Acsm2 was substantially decreased in the liver of mice fed the MCD diet, but also in haplodeficient mice fed the control diet, and to a similar extent. In turn, Acot 5 was strongly upregulated solely in $\mathrm{TNAP}^{+/-}$mice on the control diet (26-fold, Figure 6). We additionally measured the hepatic expression of hydroxy-delta-5-steroid dehydrogenase (Hds3b5), which is involved in steroid metabolism and has been found to be downregulated in steatosis. Hds 365 was virtually suppressed in $\mathrm{TNAP}^{+/-}$fed the control diet and the MCD diet (Figure 6).
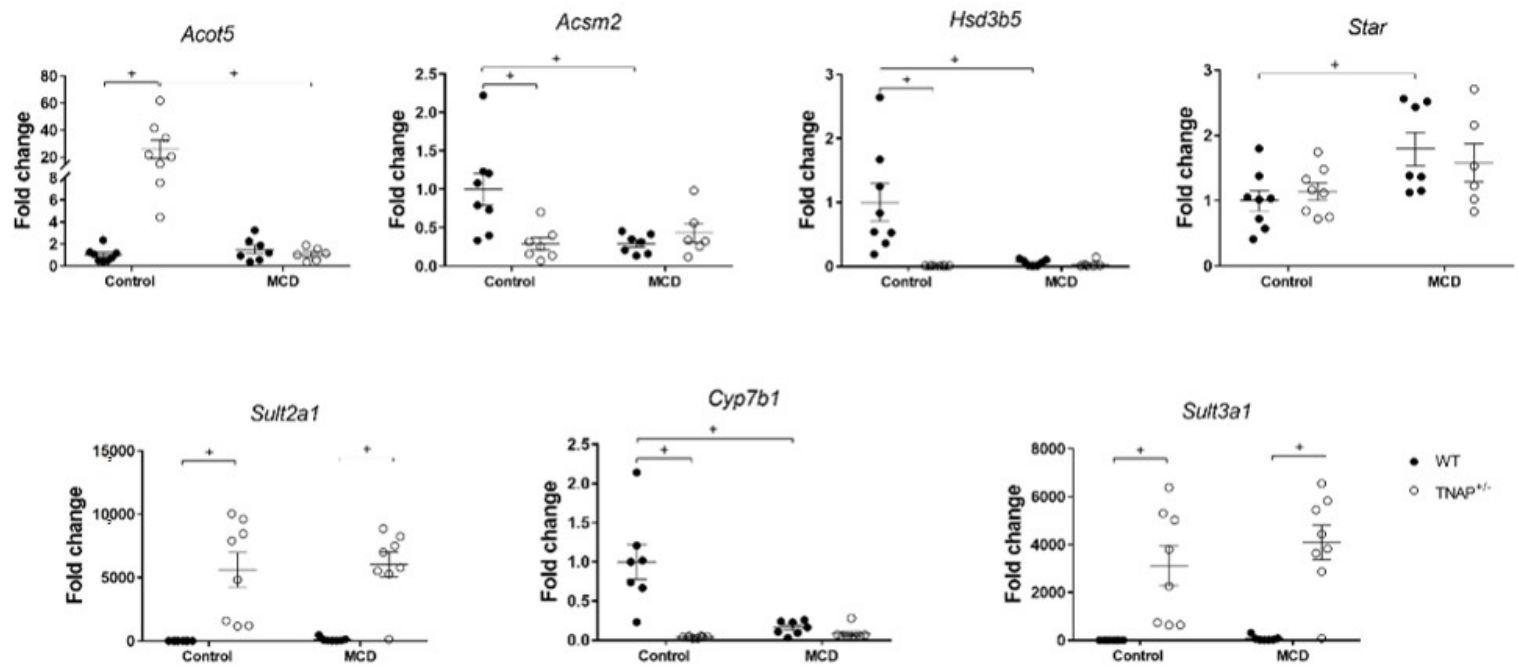

Figure 6. Hepatic expression of genes related to fatty and biliary acid metabolism in wild type (WT) and TNAP heterozygous mice $\left(\mathrm{TNAP}^{+/-}\right)$fed a control diet $\left(10 \% w / w\right.$ fat) normalized expression of qRT-PCR data are shown. ${ }^{+} p<0.05$. 
TNAP has been related to bile acid (BA) homeostasis [15]. Since steatosis has been associated with induction of the acidic BA synthetic pathway, we studied the expression of STAR (Star), 25-hydroxycholesterol 7-alpha-hydroxylase (Cyp7b1) and sulfotransferases 2a1 and 3a1 (Sulf2a1 and Sulf3a1). STAR (Steroidogenic Acute Regulatory Protein) regulates the first, rate-limiting step in the acidic BA synthetic pathway, generating oxysterols, while CYP7B1 is involved in the next step, which ultimately leads for the most part to the generation of deoxycholic acid [16]. The sulfotransferases in turn are involved in BA conjugation. Star was dramatically upregulated in all four groups receiving either the control diet or the MCD diet, more so in the latter (Figure 6 and Figure S2A). We observed a practically total suppression of Cyp $7 b 1$ expression in $\mathrm{TNAP}^{+/-}$vs. WT mice fed the control diet, comparable to that measured in WT and haplodeficient mice fed the MCD diet (Figure 6). Interestingly, our data also show augmented sulfation of BA, based on marked upregulation of Sulf2a1 and Sulf3a1, in haplodeficient mice. Notably, this profile was found to be identical in $\mathrm{TNAP}^{+/-}$mice fed the MCD diet (Figure 6).

No differences in the expression of genes involved in the synthesis of fatty acyl-CoA or bile acid metabolism were observed in mice fed the control vs. the chow diet, indicating that the alterations are due to a genotype:diet interaction (Figure S2B).

2.4. Alpl Haplodeficient Mice Exhibit a Reduced Activation of the AMPK but Not the AKT or the PPAR $\alpha$ Pathway

Because AMPK and AKT phosphorylation and the levels of PPAR $\alpha$ have been related to hepatic steatosis, Western blot analysis was carried out with liver samples of WT and TNAP haplodeficient mice fed the MCD or the control diet (Figure 7). A decreased phosphorylated to total AMPK immunoreactivity ratio was observed globally in the liver of haplodeficient mice (ANOVA $p<0.05 \mathrm{TNAP}^{+/-}$vs. WT mice irrespective of diet). However, no statistical differences were noted in AKT phosphorylation or PPAR $\alpha$ levels.
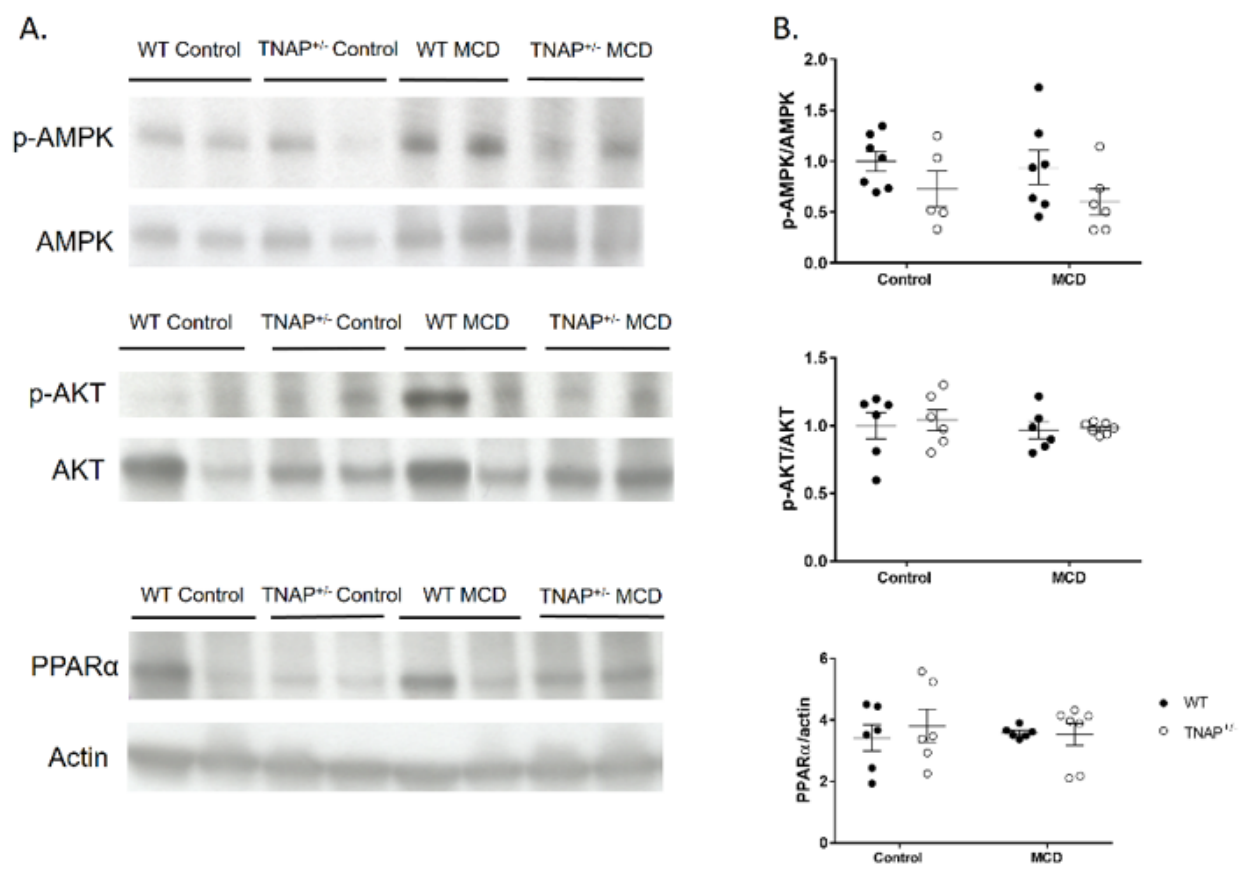

Figure 7. Hepatic ratio of p-AMPK to AMPK, p-AKT to AKT and PPAR $\alpha$ to actin in wild type (WT) and TNAP heterozygous mice (TNAP $\left.{ }^{+/}\right)$fed a control diet $(10 \% w / w$ fat, control) or the same diet deficient in choline and methionine (MCD). Western blot was used to analyze these parameters. (A) Western blot images. (B) Quantification. 


\section{Discussion}

The development of NAFLD has been connected to an increase in intestinal permeability that results in endotoxemia, inflammation and a higher fat accumulation in the liver. It has been shown that the administration of a form of alkaline phosphatase, IAP, to mice fed a high fat diet prevents the development of metabolic syndrome and the associated liver damage and steatosis [8]. These effects have been related to its inhibitory effects on fat absorption and endotoxemia [9]. Here, we studied the effect of systemic TNAP haplodeficiency in the development of NAFLD using the MCD model. The latter consists of the administration of a diet devoid of both methionine and choline, resulting in reduced hepatic mitochondrial $\beta$-oxidation and compromised very low-density lipoprotein (VLDL) synthesis [11]. Liver expression of TNAP in haplodeficient mice fed a chow diet has been previously shown to be approximately $50 \%$ of WT animals at the mRNA level, while AP activity is not substantially diminished, at least using p-nitrophenylphosphate as substrate [7]. In turn, sensitivity to levamisole is altered, consistent with a shift in the glycosylation pattern and therefore of isoform, presumably secondary to changes in biosynthetic rate $[17,18]$.

As previously described, mice fed the MCD diet lost weight progressively, an effect related to increased fatty acid mobilization in extrahepatic tissues. In fact, our experiment had to be discontinued after 17 days for ethical reasons, owing to excessive weight loss. As expected, hypoglycemia was also observed, without glucose intolerance [19]. The MCD diet induced significant fat accumulation in the liver, with no inflammation either locally or at the systemic level. In addition, no intestinal inflammation or alterations in intestinal permeability markers were observed. These data may be due to insufficient follow-up time, as TNF mRNA was already augmented in the liver of MCD-diet-fed mice, albeit nonsignificantly. In fact, our data are consistent with the observation that the MCD diet develops intestinal permeability changes after an initial phase of liver injury and TNF induction [20].

The MCD diet had very similar effects in WT and $\mathrm{TNAP}^{+/-}$mice. In turn, while WT mice fed the control diet exhibited a normal phenotype, $\mathrm{TNAP}^{+/-}$mice unexpectedly developed steatosis, which was actually comparable to that induced by the MCD diet (in either WT or haplodeficient mice). Since no liver anomalies had been previously noted in $\mathrm{TNAP}^{+/-}$mice, liver fat content of mice fed a chow diet (with a regular $4 \%$ fat content) was measured. The results obtained confirmed that haplodeficient mice do not develop steatohepatitis in these conditions, indicating that it results from the interaction of TNAP haplodeficiency with the control diet.

Despite a similar degree of hepatic steatosis, several features were different between the $\mathrm{TNAP}^{+/-}$mice fed the control diet of the MCD diet. Thus, the livers of the former presented numerous very small lipid vesicles (i.e., microsteatosis), while the livers of MCD fed mice had predominantly large lipid droplets (i.e., macrosteatosis). In addition, ALT levels were increased only in MCD-fed mice, while hepatic Il1b gene expression was upregulated exclusively in $\mathrm{TNAP}^{+/-}$mice fed the control diet (with Tnf closely following, albeit nonsignificantly, as mentioned). Similarly, Des (and Tgfb1 nonsignificantly) was increased in $\mathrm{TNAP}^{+/-}$control mice, pointing to augmented fibrosis. To gain further insight on the features of liver steatosis induced by the control diet in TNAP-deficient mice, we characterized basic genes involved in the glucidic and lipid metabolism. Data show an expression profile akin to that in the MCD diet groups. Thus Pck1, encoding cytoplasmatic PEPCK, the key enzyme in gluconeogenesis, was dramatically downregulated, consistent with inhibition of the citric acid cycle and fatty acid accumulation [21]. In contrast to other studies indicating a reduced expression of genes involved in fatty acid synthesis and desaturation [22], we found no changes in Fadsn, Acaca, Scd1, Fads1 and Fads2 in any of the groups. In order to participate in metabolic processes (oxidation and synthesis of complex lipids), fatty acids are activated by reaction with CoA to form fatty acyl CoA. This reaction is catalyzed by different fatty acid acyl-CoA synthetases, while acyl-CoA thioesterases catalyze the deactivation of fatty acid acyl-CoA. The expression of Acsm2, encoding mitochondrial acyl-coenzyme A synthetase for medium-chain fatty acids 2, 
which has been related to insulin resistance [23], was inhibited in the MCD diet groups as well as in $\mathrm{TNAP}^{+/-}$control mice, while that of Acot5, encoding an acyl-coenzyme A thioesterase, was enhanced only in $\mathrm{TNAP}^{+/-}$control mice. These data are consistent with reduced fatty acid Acyl-CoA synthesis by MCD diet and TNAP haplodeficiency with a control diet, resulting in their lipid accumulation, reinforced by enhanced deactivation of fatty acid Acyl-CoA in $\mathrm{TNAP}^{+/-} /$control mice.

Moderate elevations of serum levels of TNAP and total BA are common in NAFLD/ NASH patients [24,25]. Steatosis is associated with induction of the acidic BA biosynthetic pathway, which is normally subdued $(<10 \%)$ [16]. In addition, TNAP is induced in cholestasis, although the role of TNAP in BA homeostasis is poorly characterized [15]. Strong induction of Star, which is considered the rate-limiting step in the acidic BA pathway, was noted in all four groups receiving control/MCD diets. However, steatosis did not develop in WT mice on a control diet. The acidic pathway has been shown to lead to a smaller BA pool with increased hydrophilicity and lower cholesterol dietary absorption and improved lipid homeostasis. However, the downregulation of Cyp $7 b 1$ in this pathway, which is typically found in steatosis [26], results in the accumulation of oxysterol intermediaries, which have important inflammatory and regulatory effects. Of note, Cyp $7 b 1$ expression was strongly and inversely correlated with steatosis in the control/MCD groups. Interestingly, it was induced in WT mice on a control diet, suggesting a protective role in this context. Cyp $7 b 1$ expression is negatively regulated by insulin resistance, which was not present in our study, and also by BA stimulation of FXR [16]. The expression profile was very similar in the case of Hsd3b5, which is involved in steroid metabolism and is also downregulated in steatosis. Thus BA accumulation may be a common mechanism in $\mathrm{TNAP}^{+/-} /$control diet and MCD mice.

Interestingly, the expression of the sulfotransferases Sult2a1 and Sult3a1 was induced in TNAP haplodeficient mice fed a control diet, independently of choline and methionine content, but not with a standard diet. Sulfation is a conjugation reaction catalyzed by sulfotransferases whereby a sulfonate $\left(\mathrm{SO}_{3}{ }^{-}\right)$group is transferred from the universal sulfonate donor $3^{\prime}$-phosphoadenosine $5^{\prime}$-phosphosulfate (PAPS) to a substrate. BA sulfation is favored in cholestasis [27], and it increases renal and intestinal elimination of BA. In mice, sulfation involves the C7 position, which is less prone to hydrolysis [28]. These data are consistent with enhancement of BA elimination by TNAP haplodeficiency, a $\mathrm{TNAP}^{+/-}$/ control-diet-specific effect that cannot be ascribed to modulation of the BA acidic pathway, choline depletion or steatosis.

Our data therefore show that steatosis results from both methionine and choline deficiency and from TNAP haplodeficiency when exposed to a control diet (Figure 8). No changes were observed in mice fed the chow diet, indicating an interaction between TNAP and the control diet. The phenotype is more severe in the former, probably because the $\mathrm{MCD}$ diet produces peripheral fat mobilization, but there is clear overlap (i.e., $\mathrm{TNAP}^{+/-}$ mice are similar to WT mice). This suggests a possible common pathogenic mechanism. In this regard, TNAP may play a role in choline absorption by dephosphorylation of phosphocholine. It is possible therefore that choline deficiency may be generated in TNAP haplodeficient mice. Another possibility is the disruption of transcellular lipid transport as described for IAP KO mice in the intestine [9]. The fact that such a striking phenotype results from the lack of a single allele is remarkable, particularly considering that alkaline phosphatase activity is not significantly reduced in the liver of $\mathrm{TNAP}^{+/-}$ mice, as observed also in a previous study [7]. As these measurements have been carried out using a standard technique with p-nitrophenylphoshate as substrate, it is possible that activity varies with other substrates, due to changes in glycosylation, as stated above. It is interesting that Alpl mRNA expression was upregulated in $\mathrm{TNAP}^{+/-}$/control diet mice (despite the lack of one allele), whereas alkaline phosphatase activity was increased in both MCD groups, with different sensitivities to levamisole. Taken together, these data point to modulation of TNAP in the three steatotic groups, perhaps secondary to BA accumulation. These mechanisms will need future confirmation. 


\section{FINDINGS}

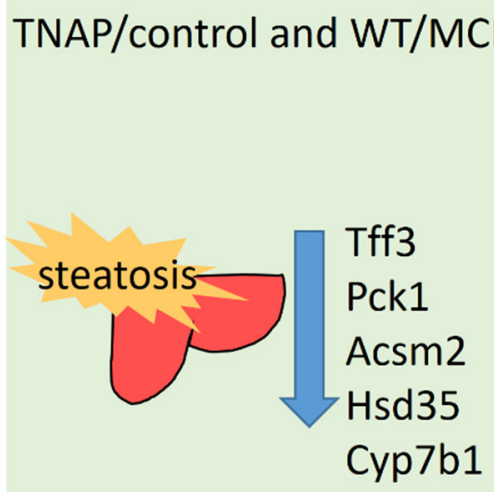

TNAP/control vs WT/MCD

Body Weight

$\mathrm{L}-1$
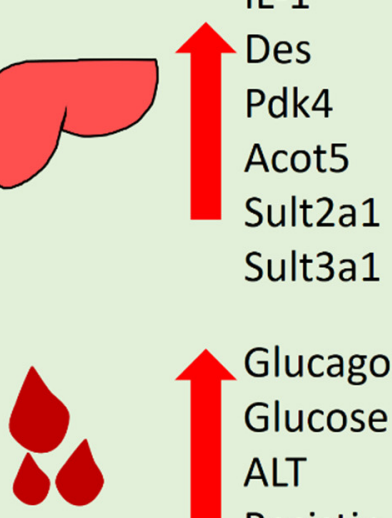

Sult3a1

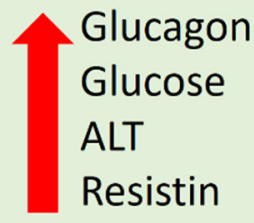

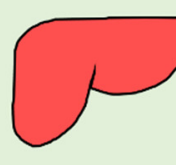

HYPOTHESIS

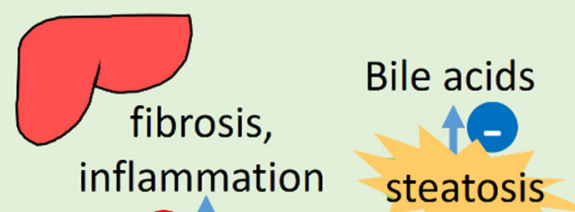

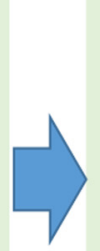
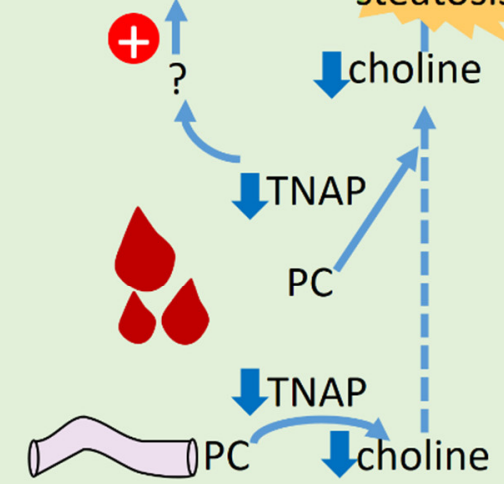

Figure 8. Hypothesis based on the findings of the study.

In conclusion, in this study, we have shown that the deficiency in TNAP associated with a control diet containing $10 \% w / w$ of fat induces a steatogenic response similar to that observed in MCD-fed WT animals, with increased inflammatory and fibrotic features and specific alterations in BA excretion.

\section{Materials and Methods}

\subsection{Animals and Experimental Design}

C57BL/ 6 heterozygous male and female mice for Alpl (B6.129S7-Akp2tm1Sor/J, referred to as $\mathrm{TNAP}^{+/-}$mice) and wild-type (WT) littermates as controls were used in the study. Seven to nine mice per group were studied. Mice were maintained at the Unit of Animal Research (Biomedical Research Center, University of Granada, Granada, Spain) in air-conditioned animal quarters with a $12 \mathrm{~h}$ light/dark cycle and specific pathogen-free conditions. Animals were housed in Makrolon ${ }^{\circledR}$ cages (4-5 mice per cage, males and females kept separately) and were given free access to autoclaved tap water and food (Harlan-Teklad 2014, Harlan Ibérica, Barcelona, Spain) until the first day of the study. Animal status was monitored daily for signs of pain or suffering. Predefined criteria for euthanasia included major weight loss, lack of movement and huddling behavior (none of the latter were detected). Mice were anesthetized with an intraperitoneal injection of a ketamine/xylazine mixture prior to cardiac exsanguination. All protocols were approved by the Animal Welfare Committee of the University of Granada (registry number: CEEA 01/03/2017-029) and carried out according to the Guide for the Care and Use of Laboratory Animals. This study complies with the requirements on reporting research detailed in the ARRIVE guidelines.

WT and $\mathrm{TNAP}^{+/-}$mice were distributed randomly to receive the MCD or the control diet (see below). Thus a $2 \times 2$ design was followed. Treatment was not blinded. Mice weights were recorded daily. Animals were fed ad libitum for 17 days, when weight loss reached the ethical limit in the MCD groups.

\subsection{Diets}

The methionine- and choline-deficient diet (Ref. TD.90262) and the control diet (Ref. TD.94149) were purchased from Envigo (Indianapolis, IN, USA). Composition is given in Tables 1 and 2. In some measurements, samples obtained from previous experiments with 
chow-fed animals were used as a reference group. After a $12 \mathrm{~h}$ fast, mice were euthanized, and plasma samples and liver tissue fragments were collected and stored at $-80^{\circ} \mathrm{C}$.

Table 1. Composition of diets.

\begin{tabular}{cccc}
\hline & Control & MCD Diet & Chow Diet \\
\hline Sucrose $(\mathrm{g} / \mathrm{kg})$ & 455 & 455 & 445 \\
Corn starch $(\mathrm{g} / \mathrm{kg})$ & 200 & 200 & 200 \\
Corn Oil $(\mathrm{g} / \mathrm{kg})$ & 100 & 100 & 40 \\
1-Methionine $(\mathrm{g} / \mathrm{kg})$ & 8.2 & - & 8.2 \\
Choline $(\mathrm{g} / \mathrm{kg})$ & 1.4 & - & 1.4 \\
\hline
\end{tabular}

Table 2. Elemental composition of MCD and control diets.

\begin{tabular}{|c|c|c|}
\hline $\mathrm{g} / \mathrm{Kg}$ & Control Diet & MCD Diet \\
\hline Sucrose & 443.597 & 455.294 \\
\hline Corn starch & 198.783 & 200.0 \\
\hline Corn Oil & 100.0 & 100.0 \\
\hline Cellulose & 30.0 & 30.0 \\
\hline Mineral Mix, AIN-76 (170915) & 35.0 & 35.0 \\
\hline Calcium Phosphate, dibasic & 3.0 & 3.0 \\
\hline L-Alanine & 3.5 & 3.5 \\
\hline L-Arginine $\mathrm{HCl}$ & 12.1 & 12.1 \\
\hline L-Asparagine & 6.0 & 6.0 \\
\hline L-Aspartic Acid & 3.5 & 3.5 \\
\hline L-Cysteine & 3.5 & 3.5 \\
\hline L-Glutamic Acid & 40.0 & 40.0 \\
\hline Glycine & 23.3 & 23.3 \\
\hline L-Histidine $\mathrm{HCl}$, monohydrate & 4.5 & 4.5 \\
\hline L-Isoleucine & 8.2 & 8.2 \\
\hline L-Leucine & 11.1 & 11.1 \\
\hline L-Lysine $\mathrm{HCl}$ & 18.0 & 18.0 \\
\hline L-Methionine & 8.2 & - \\
\hline L-Phenylalanine & 7.5 & 7.5 \\
\hline L-Proline & 3.5 & 3.5 \\
\hline L-Serine & 3.5 & 3.5 \\
\hline L-Threonine & 8.2 & 8.2 \\
\hline L-Tryptophan & 1.8 & 1.8 \\
\hline L-Tyrosine & 5.0 & 5.0 \\
\hline L-Valine & 8.2 & 8.2 \\
\hline Vitamin Mix, Teklad (400600) & 10.0 & \\
\hline + Choline dihydrogen citrate & $(1.4)$ & - \\
\hline Vitamin Mix, w/o choline, A, D, E (83171) & - & 5.0 \\
\hline Vitamin E, DL- $\alpha$ tocopherol acetate $(500 \mathrm{IU} / \mathrm{g}$ ) & - & 0.242 \\
\hline Vitamin A Palmitate $(500,000 \mathrm{IU} / \mathrm{g})$ & - & 0.0396 \\
\hline Vitamin D3, cholecalciferol (500,000 IU/g) & - & 0.0044 \\
\hline Ethoxyquin, antioxidant & 0.02 & 0.02 \\
\hline
\end{tabular}

\subsection{Histological Assessment}

Liver tissue fragments were fixed in $4 \%$ paraformaldehyde and embedded in paraffin. For histological evaluation, slides were stained with hematoxylin and eosin (H\&E). Images were taken and digitalized using a Leica DMI3000B microscope (Leica, Wetzlar, Germany) equipped with a Leica DFC420 camera. Sections were scored blindly for fat-drops size and frequency.

\subsection{Plasmatic Parameters}

Fasting plasma samples were assayed for alanine aminotransferase activity (SigmaAldrich, San Luis, MO, USA) and glucose concentration. The glucose oxidase method 
was used. Plasmatic parameters were measured using Metabolic Hormone Expanded Panel multiplex (ref. MMHE-44K, Merk Millipore, Burlington, MA, USA) according to manufacturer's instructions. HOMA-IR was calculated using the formula:

$$
H O M A-I R=\frac{g l u c o s e\left(\frac{m g}{d L}\right) \times \text { insulin }}{405}
$$

\subsection{Alkaline Phosphatase Activity}

Colonic and hepatic tissues were mechanically homogenized in HTAB (Hexadecyltrimethylammonium Bromide) buffer using a Bullet Blender (Next Advance, Averill Park, NW, USA). Alkaline phosphatase activity was measured spectrophotometrically, using $5.5 \mathrm{mM}$ disodium nitrophenyl phosphate as substrate. Sensitivity to the specific inhibitor levamisole was tested and expressed as inhibition percentage.

\subsection{Hepatic Fat Content}

Liver samples were homogenized in distilled water using an electric homogenizer (Heidolph, Schwabach, Germany). Chloroform:methanol solution was added to the homogenates to a 1:1:1 mix that was vortexed and centrifuged at $1100 \mathrm{~g} / 20 \mathrm{~min} / 4{ }^{\circ} \mathrm{C}$. The organic phase was collected and dried under a stream of nitrogen. Dry extract was weighed, and results were reported as $\mathrm{mg}$ fat per gram of liver.

\subsection{RNA Isolation and Quantitative Reverse-Transcription Polymerase Chain Reaction (RT-qPCR) Analysis}

RNA was isolated using RNeasy MiniKit (Qiagen, CA, USA) following the manufacturer's instructions. RNA quantity and integrity were assessed spectrophotometrically with Nanodrop (Thermo Fisher Scientific, Waltham, MA, USA). Total RNA was subjected to reverse transcription (BioRad, Hercules, CA, USA), and resulting cDNA was subjected to quantitative PCR (Promega, Madison, WI, USA). Primers used to amplify Srebf1, Fasn and Acaca were predesign primers from MilliporeSigma (Madrid, Spain). Other primers used, and also purchased from MilliporeSigma, are shown in Table S1.

\subsection{Western Blot}

Liver tissues were homogenized in lysis buffer $(0.1 \% w / v$ SDS, $0.1 \% w / v$ sodium deoxycholate, $1 \% v / v$ Triton X-100 in PBS) with a protease inhibitor cocktail 1:200 $(v / v)(\mathrm{P} 9599$; MilliporeSigma, Madrid, Spain) and a phosphatase inhibitor cocktail 1:100 (v/v) (SC-45045; Santa Cruz Biotechnology, Dallas, TX, USA) using a Bullet Blender (Next Advance, Averill Park, NW, USA). Homogenates were sonicated and centrifuged $12,000 \mathrm{~g} / 10 \mathrm{~min} / 4{ }^{\circ} \mathrm{C}$. After protein concentration was determined by the bicinchoninic acid assay [29], samples were heated at $95^{\circ} \mathrm{C}$ for $5 \mathrm{~min}$ in Laemmli sample buffer (BioRad, Hercules, CA, USA). Proteins were separated by SDS-PAGE and transferred onto nitrocellulose membranes. Samples were immunoblotted using the primary antibodies rabbit anti-AKT (ref. 9272s, Cell Signaling Technology, MA, USA), Ser473 rabbit anti-pAKT antibody (ref. 4060s, Cell Signaling Technology, MA, USA), rabbit anti-AMPK $\alpha$ antibody (ref. 2532L, Cell Signaling Technology, MA, USA), Thr172 rabbit anti-pAMPK $\alpha$ antibody (ref. 2535s, Cell Signaling Technology, MA, USA), rabbit anti-PPAR $\alpha$ antibody (ref. ab3484, Abcam, Cambridge, UK) and mouse anti-actin (A5441, Sigma-Aldrich, San Luis, MO, USA) at 1:1000 dilution. After incubation with a secondary antibody, bands were detected by ECL (PerkinElmer, Waltham, MA, USA) and quantified using ImageJ (National Institutes of Health, USA).

\subsection{Statistical Analysis}

Samples were run at least in triplicate, and results were expressed as mean \pm standard error of the mean (SEM). Differences among means were tested by two-way ANOVA and a posteriori Tukey's multiple comparisons test or Student's $t$-test for pairwise comparisons. Analyses were carried out with GraphPad Prism 7 software (GraphPad Software, San Diego CA, USA). Significance was accepted at $p \leq 0.05$. 
Supplementary Materials: Supplementary materials can be found at https:/ /www.mdpi.com/1422 $-0067 / 22 / 1 / 51 / s 1$.

Author Contributions: Conceptualization, F.S.d.M. and O.M.-A.; investigation, M.T.-G., R.G.-B., C.H.-C., S.C. and D.C.-H.; original draft, R.G.-B. and M.T.-G.; review and editing of the manuscript, all authors; data analysis, R.G.-B. and M.T.-G.; All authors have read and agreed to the published version of the manuscript.

Funding: This research was funded by the Ministry of Economy and Competitivity of Spain, partly with Fondo Europeo de Desarrollo Regional FEDER funds [BFU2014-57736-P, AGL2014-58883-R, SAF2017-88457-R, AGL2017-85270-R] and by Junta de Andalucía [CTS235, CTS164]. MTG, RGB and $\mathrm{CHC}$ were supported by fellowships from the Ministry of Education. CIBERehd is funded by Instituto de Salud Carlos III.

Institutional Review Board Statement: The study was conducted according to the guidelines of the Guide for the Care and Use of Laboratory Animals, and approved by the Animal Welfare Committee of the University of Granada (registry number: CEEA 01/03/2017-029).

Informed Consent Statement: Not applicable for studies not involving humans.

Acknowledgments: We gratefully acknowledge the assistance of Mercedes González and the rest of the group.

Conflicts of Interest: Authors declare no conflict of interest.

$\begin{array}{ll}\text { Abbreviations } \\ \text { ALT } & \begin{array}{l}\text { alanine aminotransferase } \\ \text { bile acids }\end{array} \\ \text { BA } & \text { gastric inhibitory peptide } \\ \text { GIP } & \text { glucagon-like peptide } 1 \\ \text { GLP-1 } & \text { homeostasis model assessment-insulin resistance } \\ \text { HOMA-IR } & \text { intestinal alkaline phosphatase } \\ \text { IAP } & \text { Methionine- and choline- deficient diet } \\ \text { MCD } & \text { nonalcoholic fatty liver disease } \\ \text { NAFLD } & \text { nonalcoholic steatohepatitis } \\ \text { NASH } & \text { Phosphorylcholine } \\ \text { PC } & \text { pancreatic peptide } \\ \text { PAP } & \text { phosphoenolpyruvate carboxykinase } \\ \text { PEPCK } & \text { peptide YY } \\ \text { PYY } & \text { tissue nonspecific alkaline phosphatase } \\ \text { TNAP } & \text { very low-density lipoproteins }\end{array}$

\section{References}

1. Raza, S.; Rajak, S.; Upadhyay, A.; Tewari, A.; Anthony Sinha, R. Current treatment paradigms and emerging therapies for NAFLD/NASH. Front. Biosci. 2021, 26, 206-237. [CrossRef]

2. Pafili, K.; Roden, M. Non-alcoholic fatty liver disease (NAFLD) from pathogenesis to treatment concepts in humans. Mol. Metab. 2020, 101122. [CrossRef] [PubMed]

3. Rader, B.A. Alkaline Phosphatase, an Unconventional Immune Protein. Front. Immunol. 2017, 8, 897. [CrossRef]

4. Buchet, R.; Millan, J.L.; Magne, D. Multisystemic functions of alkaline phosphatases. Methods Mol. Biol. 2013 , 1053, $27-51$.

5. Gamez-Belmonte, R.; Hernandez-Chirlaque, C.; Sanchez de Medina, F.; Martinez-Augustin, O. Experimental acute pancreatitis is enhanced in mice with tissue nonspecific alkaline phoshatase haplodeficiency due to modulation of neutrophils and acinar cells. Biochim. Biophys. Acta Mol. Basis Dis. 2018, 1864, 3769-3779. [CrossRef]

6. Bessueille, L.; Briolay, A.; Como, J.; Mebarek, S.; Mansouri, C.; Gleizes, M.; El Jamal, A.; Buchet, R.; Dumontet, C.; Matera, E.L.; et al. Tissue-nonspecific alkaline phosphatase is an anti-inflammatory nucleotidase. Bone 2020, 133, 115262. [CrossRef] [PubMed]

7. Hernandez-Chirlaque, C.; Gamez-Belmonte, R.; Ocon, B.; Martinez-Moya, P.; Wirtz, S.; Sanchez de Medina, F.; Martinez-Augustin, O. Tissue Non-specific Alkaline Phosphatase Expression is Needed for the Full Stimulation of T Cells and T Cell-Dependent Colitis. J. Crohn's Colitis 2017, 11, 857-870.

8. Kaliannan, K.; Hamarneh, S.R.; Economopoulos, K.P.; Nasrin Alam, S.; Moaven, O.; Patel, P.; Malo, N.S.; Ray, M.; Abtahi, S.M.; Muhammad, N.; et al. Intestinal alkaline phosphatase prevents metabolic syndrome in mice. Proc. Natl. Acad. Sci. USA 2013, 110, 7003-7008. [CrossRef] 
9. Nakano, T.; Inoue, I.; Koyama, I.; Kanazawa, K.; Nakamura, K.-I.; Narisawa, S.; Tanaka, K.; Akita, M.; Masuyama, T.; Seo, M.; et al. Disruption of the murine intestinal alkaline phosphatase gene Akp3 impairs lipid transcytosis and induces visceral fat accumulation and hepatic steatosis. Am. J. Physiol. Gastrointest. Liver Physiol. 2007, 292, G1439-G1449. [CrossRef] [PubMed]

10. Sherriff, J.L.; O'Sullivan, T.A.; Properzi, C.; Oddo, J.L.; Adams, L.A. Choline, Its Potential Role in Nonalcoholic Fatty Liver Disease, and the Case for Human and Bacterial Genes. Adv. Nutr. 2016, 7, 5-13. [CrossRef]

11. Rinella, M.E.; Elias, M.S.; Smolak, R.R.; Fu, T.; Borensztajn, J.; Green, R.M. Mechanisms of hepatic steatosis in mice fed a lipogenic methionine choline-deficient diet. J. Lipid Res. 2008, 49, 1068-1076. [CrossRef] [PubMed]

12. Bin, P.; Huang, R.; Zhou, X. Oxidation Resistance of the Sulfur Amino Acids: Methionine and Cysteine. BioMed Res. Int. 2017, 2017, 9584932. [CrossRef] [PubMed]

13. Willebrords, J.; Maes, M.; Pereira, I.V.A.; da Silva, T.C.; Govoni, V.M.; Lopes, V.V.; Crespo Yanguas, S.; Shestopalov, V.I.; Nogueira, M.S.; de Castro, I.A.; et al. Protective effect of genetic deletion of pannexin1 in experimental mouse models of acute and chronic liver disease. Biochim. Biophys. Acta Mol. Basis Dis. 2018, 1864, 819-830. [CrossRef]

14. Bujak, M.; Bujak, I.T.; Sobočanec, S.; Mihalj, M.; Novak, S.; Ćosić, A.; Levak, M.T.; Kopačin, V.; Mihaljević, B.; Balog, T.; et al. Trefoil Factor 3 Deficiency Affects Liver Lipid Metabolism. Cell. Physiol. Biochem. 2018, 47, 827-841. [CrossRef] [PubMed]

15. Poupon, R. Liver alkaline phosphatase: A missing link between choleresis and biliary inflammation. Hepatology 2015, 61, 2080-2090. [CrossRef] [PubMed]

16. Pandak, W.M.; Kakiyama, G. The acidic pathway of bile acid synthesis: Not just an alternative pathway. Liver Res. 2019, 3, 88-98. [CrossRef]

17. Lopez-Posadas, R.; Gonzalez, R.; Ballester, I.; Martinez-Moya, P.; Romero-Calvo, I.; Suarez, M.D.; Zarzuelo, A.; Martinez-Augustin, O.; Sanchez de Medina, F. Tissue-nonspecific alkaline phosphatase is activated in enterocytes by oxidative stress via changes in glycosylation. Inflamm. Bowel. Dis. 2011, 17, 543-556. [CrossRef]

18. Sanchez de Medina, F.; Martinez-Augustin, O.; Gonzalez, R.; Ballester, I.; Nieto, A.; Galvez, J.; Zarzuelo, A. Induction of alkaline phosphatase in the inflamed intestine: A novel pharmacological target for inflammatory bowel disease. Biochem. Pharmacol. 2004, 68, 2317-2326. [CrossRef]

19. Machado, M.V.; Michelotti, G.A.; Xie, G.; Almeida Pereira, T.; Boursier, J.; Bohnic, B.; Guy, C.D.; Diehl, A.M. Mouse models of diet-induced nonalcoholic steatohepatitis reproduce the heterogeneity of the human disease. PLoS ONE 2015, 10, e0127991. [CrossRef]

20. Luther, J.; Garber, J.J.; Khalili, H.; Dave, M.; Bale, S.S.; Jindal, R.; Motola, D.L.; Luther, S.; Bohr, S.; Jeoung, S.W.; et al. Hepatic Injury in Nonalcoholic Steatohepatitis Contributes to Altered Intestinal Permeability. Cell. Mol. Gastroenterol. Hepatol. 2015, 1, 222-232.e2. [CrossRef]

21. Burgess, S.C.; Hausler, N.; Merritt, M.; Jeffrey, F.M.; Storey, C.; Milde, A.; Koshy, S.; Lindner, J.; Magnuson, M.A.; Malloy, C.R.; et al. Impaired tricarboxylic acid cycle activity in mouse livers lacking cytosolic phosphoenolpyruvate carboxykinase. J. Biol. Chem. 2004, 279, 48941-48949. [CrossRef] [PubMed]

22. Jha, P.; Knopf, A.; Koefeler, H.; Mueller, M.; Lackner, C.; Hoefler, G.; Claudel, T.; Trauner, M. Role of adipose tissue in methioninecholine-deficient model of non-alcoholic steatohepatitis (NASH). Biochim. Biophys. Acta 2014, 1842, 959-970. [CrossRef] [PubMed]

23. Boomgaarden, I.; Vock, C.; Klapper, M.; Döring, F. Comparative analyses of disease risk genes belonging to the acyl-CoA synthetase medium-chain (ACSM) family in human liver and cell lines. Biochem. Genet. 2009, 47, 739-748. [CrossRef] [PubMed]

24. Bechmann, L.P.; Kocabayoglu, P.; Sowa, J.P.; Sydor, S.; Best, J.; Schlattjan, M.; Beilfuss, A.; Schmitt, J.; Hannivoort, R.A.; Kilicarslan, A.; et al. Free fatty acids repress small heterodimer partner (SHP) activation and adiponectin counteracts bile acid-induced liver injury in superobese patients with nonalcoholic steatohepatitis. Hepatology 2013, 57, 1394-1406. [CrossRef] [PubMed]

25. Chen, J.; Deng, W.; Wang, J.; Shao, Y.; Ou, M.; Ding, M. Primary bile acids as potential biomarkers for the clinical grading of intrahepatic cholestasis of pregnancy. Int. J. Gynaecol. Obstet. 2013, 122, 5-8. [CrossRef] [PubMed]

26. Guillén, N.; Navarro, M.A.; Arnal, C.; Noone, E.; Arbonés-Mainar, J.M.; Acín, S.; Surra, J.C.; Muniesa, P.; Roche, H.M.; Osada, J. Microarray analysis of hepatic gene expression identifies new genes involved in steatotic liver. Physiol. Genom. 2009, 37, 187-198. [CrossRef] [PubMed]

27. Dawson, P.A.; Setchell, K.D.R. Will the real bile acid sulfotransferase please stand up? Identification of Sult2a8 as a major hepatic bile acid sulfonating enzyme in mice. J. Lipid. Res. 2017, 58, 1033-1035. [CrossRef]

28. Robben, J.; Parmentier, G.; Eyssen, H. Isolation of a rat intestinal Clostridium strain producing 5 alpha- and 5 beta-bile salt 3 alpha-sulfatase activity. Appl. Environ. Microbiol. 1986, 51, 32-38. [CrossRef]

29. Smith, P.K.; Krohn, R.I.; Hermanson, G.T.; Mallia, A.K.; Gartner, F.H.; Provenzano, M.D.; Fujimoto, E.K.; Goeke, N.M.; Olson, B.J.; Klenk, D.C. Measurement of protein using bicinchoninic acid. Anal. Biochem. 1985, 150, 76-85. [CrossRef] 